\title{
Chaotic transitions and low-frequency fluctuations in semiconductor lasers with optical feedback
}

\author{
Ruslan L. Davidchack $^{\mathrm{a}, *}$, Ying-Cheng Lai ${ }^{\mathrm{a}, 1}$, Athanasios Gavrielides ${ }^{\mathrm{b}}$, \\ Vassilios Kovanis ${ }^{\mathrm{b}}$ \\ ${ }^{a}$ Department of Physics and Astronomy, University of Kansas, Lawrence, KS 66045, USA \\ b Nonlinear Optics Group, Air Force Research Laboratory DELO, Kirtland AFB, NM 87117, USA \\ Received 5 March 1999; received in revised form 19 April 2000; accepted 21 April 2000 \\ Communicated by C.K.R.T. Jones
}

\begin{abstract}
This paper examines the dynamical origin of low-frequency fluctuations (LFFs) in semiconductor lasers subject to timedelayed optical feedback. In particular, we study chaotic transitions leading to the onset of LFFs by numerical integration of Lang-Kobayashi equations for a laser pumped near threshold. We construct a bifurcation analysis scheme that enables the classification of the different operation regimes of the laser. We use the scheme to study the coexistence of the LFFs and stable emission on the maximum gain mode (MGM), which was the subject of recent experiments [T. Heil, I. Fischer, W. Elsäßer, Phys. Rev. A 60 (1999) 634]. Our computations suggest that as the feedback level increases, the regime of sustained LFFs alternates with regions of transient LFFs, where the laser can achieve stabilization on the MGM. Exploration of the parameter space reveals strong dependence of the structure of the LFF dynamics and the coexistence regime on the value of the linewidth enhancement factor. () 2000 Elsevier Science B.V. All rights reserved.
\end{abstract}

PACS: 05.45.-a; 42.55.Px; 42.65.Sf

Keywords: Lang-Kobayashi equations; Poincaré sections; Transient chaos

\section{Introduction}

Semiconductor lasers are of tremendous value to a large variety of applications including digital communication. In a typical application, the presence of small optical feedback is inevitable. The feedback can be from the end mirrors of the laser cavity itself, or it can come from reflections off other optical components in the system. As such, the feedback is typically time-delayed. Dynamically, semiconductor lasers subject to optical feedback are then described by differential equations with a time-delay. If one intends to model such a system by using first-order autonomous differential equations, one finds that an infinite number of them is required. The phase-space dimension of the

\footnotetext{
* Corresponding author.

E-mail address: ruslan@ukans.edu (R.L. Davidchack).

${ }^{1}$ Present address: Department of Mathematics and Electrical Engineering, Center for Systems Science and Engineering Research, Arizona State University, Tempe, AZ 85287, USA.
} 
model is thus infinite. Although in certain cases the asymptotic dynamics, or attractors, may be low-dimensional, analysis and numerical computation of delay differential equations still presents challenging problems in nonlinear dynamics.

The aim of this paper is to study one phenomenon of semiconductor lasers with optical feedback which is commonly observed in experiments at moderate levels of feedback strength when biased close to solitary threshold. The phenomenon is called low-frequency fluctuations (LFFs) in the power output of the laser. In particular, it has been known for more than two decades that the power of such a laser can typically exhibit dropouts, even down to zero, at somewhat irregular time intervals [1]. The average frequency of the power dropouts is much smaller than that of the solitary laser relaxation oscillations or the external-cavity modes (ECMs) (hence the term "LFFs"). The power fluctuations are quite annoying in practical applications and, therefore, it becomes important to study the mechanism for these fluctuations. There has been tremendous amount of work on LFFs in semiconductor lasers [2-12] targeting different aspects of the phenomenon. One important recent observation is that in some regions of the parameter space the LFFs coexist with stable emission on the maximum gain mode (MGM) [13,14]. In such cases it may be speculated that the LFFs are only a transient phenomenon [13]. That is, the laser output can become stable after a (long) period of initial transient time. If this were true, then LFFs would not be a serious problem in practice.

The main question we address in this paper is: How prevalent is the transiency of the LFF phenomenon? In order to answer this question, a good understanding of the global properties of the underlying chaotic invariants is required. Since analytic study of nonlinear systems generally reveals only local properties (even in the case of simple low-dimensional systems), investigation by means of numerical modeling becomes essential. Our approach is to perform a detailed numerical bifurcation analysis in order to understand LFFs from the perspective of chaotic dynamics. Our principal result is that LFFs can be either transient or sustained, depending on the basin structure of coexisting attractors that physically correspond to different operational modes of the laser. In particular, when the laser is pumped near the lasing threshold of a solitary laser, the value of the linewidth enhancement factor $\alpha$ $[15,16]$ strongly influences the operation regime of the laser. For small $\alpha$, the laser settles into a stable operation on the MGM for all levels of the delayed optical feedback, while for large $\alpha$, the laser operates in a sustained LFF regime, which also persists for all levels of feedback. However, in the intermediate range of $\alpha$ values, the regime of sustained LFFs alternates with "windows" of transient LFFs, where the laser eventually stabilizes at the MGM [34].

The rest of the paper is organized as follows. In Section 2 we describe the Lang-Kobayashi equations [17], which have been established to be the fundamental equations for semiconductor lasers subject to optical feedback. System evolution at different feedback levels obtained from the numerical solution of Lang-Kobayashi equations is described in Section 3. In Section 4 we construct a two-dimensional Poincaré surface of section passing through all the stationary solutions, which is particularly useful for analyzing the chaotic transitions and the phenomenon of LFFs. Results of the bifurcation analysis which lead to our principal conclusion about the dynamical nature of the LFFs are presented in Section 5. We conclude with a discussion in Section 6.

\section{Lang-Kobayashi equations}

The dynamics of semiconductor lasers subject to optical feedback is described by the Lang-Kobayashi equations [17] for the intracavity complex electric field $\mathcal{E}(t)$ and the carrier population $N(t)$, as follows:

$$
\begin{aligned}
\frac{\mathrm{d} \mathcal{E}(t)}{\mathrm{d} t} & =\frac{1}{2}(1+\mathrm{i} \alpha)\left(G(N, \mathcal{E})-\tau_{\mathrm{p}}^{-1}\right) \mathcal{E}(t)+\gamma \exp \left(-\mathrm{i} \omega_{0} \tau\right) \mathcal{E}(t-\tau), \\
\frac{\mathrm{d} N(t)}{\mathrm{d} t} & =J-\frac{N(t)}{\tau_{\mathrm{s}}}-G(N, \mathcal{E})|\mathcal{E}(t)|^{2},
\end{aligned}
$$


where $\alpha$ is the linewidth enhancement factor $[15,16], \omega_{0}$ the laser frequency without feedback, $\tau_{\mathrm{p}}$ the photon lifetime, $\tau_{\mathrm{s}}$ the carrier lifetime, and $J$ is the injected current density. The external-cavity parameters are the feedback parameter $\gamma$ that measures the amount of the light reflected back into the laser cavity, and the delay time $\tau=2 L_{\text {ext }} / c$, which is the round-trip time of the light in the external cavity of length $L_{\mathrm{ext}}$. This model assumes single-mode operation and neglects multiple reflections. Despite recent discussions about the importance of multimode behavior for the description of LFFs [11], we believe this model remains suitable for our study, since in the experiments the LFFs persist even when an etalon is inserted, forcing the laser to operate in single-mode [14]. Also, in order to isolate purely deterministic properties of our analysis, we neglect the effect of spontaneous emission noise, which is not crucial for the occurrence of the LFFs, even though it can significantly influence the statistics of the dropout events [9].

The modal gain per unit time $G(N, \mathcal{E})=G_{0}(N)\left(1-\varepsilon \mathcal{E}^{2}\right)$ contains the linear gain $G_{0}(N)=G_{N}\left(N-N_{0}\right)$, where $G_{N}$ is the gain constant and $N_{0}$ is the carrier density at transparency, and intensity reduction of the gain due to spatial and spectral hole burning and carrier heating, with $\varepsilon$ being the gain saturation coefficient $[18,19]$. The effect of nonlinear gain saturation was extensively studied by Masoller [19] and was found to lead to the stabilization of the laser dynamics, so that the onset of chaos and chaotic transitions is shifted towards higher feedback levels. Since the purpose of this work is to investigate chaotic transitions in the system with delayed feedback, we will ignore the saturation effects and set $\varepsilon=0$. The electric field is normalized so that $V_{\mathrm{c}}|\mathcal{E}(t)|^{2}$ is the total photon number in the laser wave guide, where $V_{\mathrm{c}}$ is the volume of the active region. The parameter $J_{\mathrm{th}}=N_{\mathrm{th}} / \tau_{\mathrm{s}}$ is the lasing threshold current density of a solitary laser and $N_{\text {th }}$ is the threshold carrier density.

For the sake of numerical convenience, we measure time in units of the photon lifetime and introduce the normalized excess carrier number density $n(t) \sim N(t)-N_{\text {th }}$. We rewrite the Lang-Kobayashi equations in the following normalized form [20]:

$$
\frac{\mathrm{d} \mathcal{E}(t)}{\mathrm{d} t}=(1+\mathrm{i} \alpha) n(t) \mathcal{E}(t)+\eta \exp \left(-\mathrm{i} \omega_{0} \tau\right) \mathcal{E}(t-\tau), \quad T \frac{\mathrm{d} n(t)}{\mathrm{d} t}=P-n(t)-(2 n(t)+1)|\mathcal{E}(t)|^{2},
$$

where $\eta=\tau_{\mathrm{p}} \gamma, T=\tau_{\mathrm{s}} / \tau_{\mathrm{p}}$, and $P \sim J-J_{\text {th }}$. The above equations describe dynamics of three independent real variables, since $\mathcal{E}(t)=\mathcal{E}_{\mathrm{R}}(t)+\mathrm{i} \mathcal{E}_{\mathrm{I}}(t)$. To integrate these equations starting at $t=0$, it is necessary to define initial conditions for the three variables everywhere in the time interval $-\tau \leq t<0$.

While analytic studies of Eq. (2) are infeasible at present, the stationary solutions (fixed points) and their stability can still be obtained. One can readily see that the system allows for the stationary solutions in the form

$$
\mathcal{E}(t)=E_{\mathrm{s}} \exp \left(\mathrm{i}\left(\omega_{\mathrm{s}}-\omega_{0}\right) t\right) \quad \text { and } \quad n(t)=n_{\mathrm{s}} .
$$

If we write the complex electric field $\mathcal{E}(t)$ in the form

$$
\mathcal{E}(t)=E(t) \exp \left(\mathrm{i}\left(\phi(t)-\omega_{0} t\right)\right)
$$

and introduce the variable $\Delta(t)=\phi(t)-\phi(t-\tau)$, which is the phase delay during the external-cavity round-trip time $\tau$, then the stationary solutions are the fixed points in the configuration space of the three variables $E(t), n(t)$, and $\Delta(t)$. Substitution of the stationary solutions into Eq. (2) yields

$$
n_{\mathrm{s}}=-\eta \cos \Delta_{\mathrm{s}}
$$

and

$$
E_{\mathrm{s}}^{2}=\frac{P-n_{\mathrm{s}}}{1+2 n_{\mathrm{s}}} \geq 0
$$

where $\Delta_{\mathrm{S}} \equiv \omega_{\mathrm{s}} \tau$ are the solutions to the implicit equation

$$
\Delta_{\mathrm{S}}-\omega_{0} \tau=-\eta \tau\left(\alpha \cos \Delta_{\mathrm{S}}+\sin \Delta_{\mathrm{S}}\right)=-\eta \tau\left(\alpha^{2}+1\right)^{1 / 2} \sin \left(\Delta_{\mathrm{S}}+\arctan \alpha\right) .
$$


At low feedback levels this equation has only one solution, which is close to the solitary laser frequency $\omega_{0}$. With increasing $\eta$, additional solutions appear in pairs. The feedback level at which a new pair of fixed point solutions is created can be determined by approximating the extrema of the RHS of Eq. (7) with parabolas, resulting in the following formula:

$$
\eta \approx \frac{\beta+\left(\beta^{2}-2\right)^{1 / 2}}{2 \tau\left(\alpha^{2}+1\right)^{1 / 2}}
$$

where $\beta=\omega_{0} \tau+\arctan \alpha+\left(2 n-\frac{1}{2}\right) \pi, n=1,2, \ldots$. The approximation improves with increasing $n$.

A stability analysis [21] shows that one of the points is inherently stable and is thus identified with the ECM of the laser, while the other is the saddle-point instability, often called an antimode, which physically corresponds to destructive interference between the external cavity and laser fields [8]. The stability condition is given by

$$
1-\eta \tau\left(\alpha \sin \Delta_{\mathrm{s}}-\cos \Delta_{\mathrm{s}}\right)>0
$$

Because of the condition $E_{\mathrm{s}} \geq 0$, some of the solutions of Eq. (7) are not allowed when $P<\eta$.

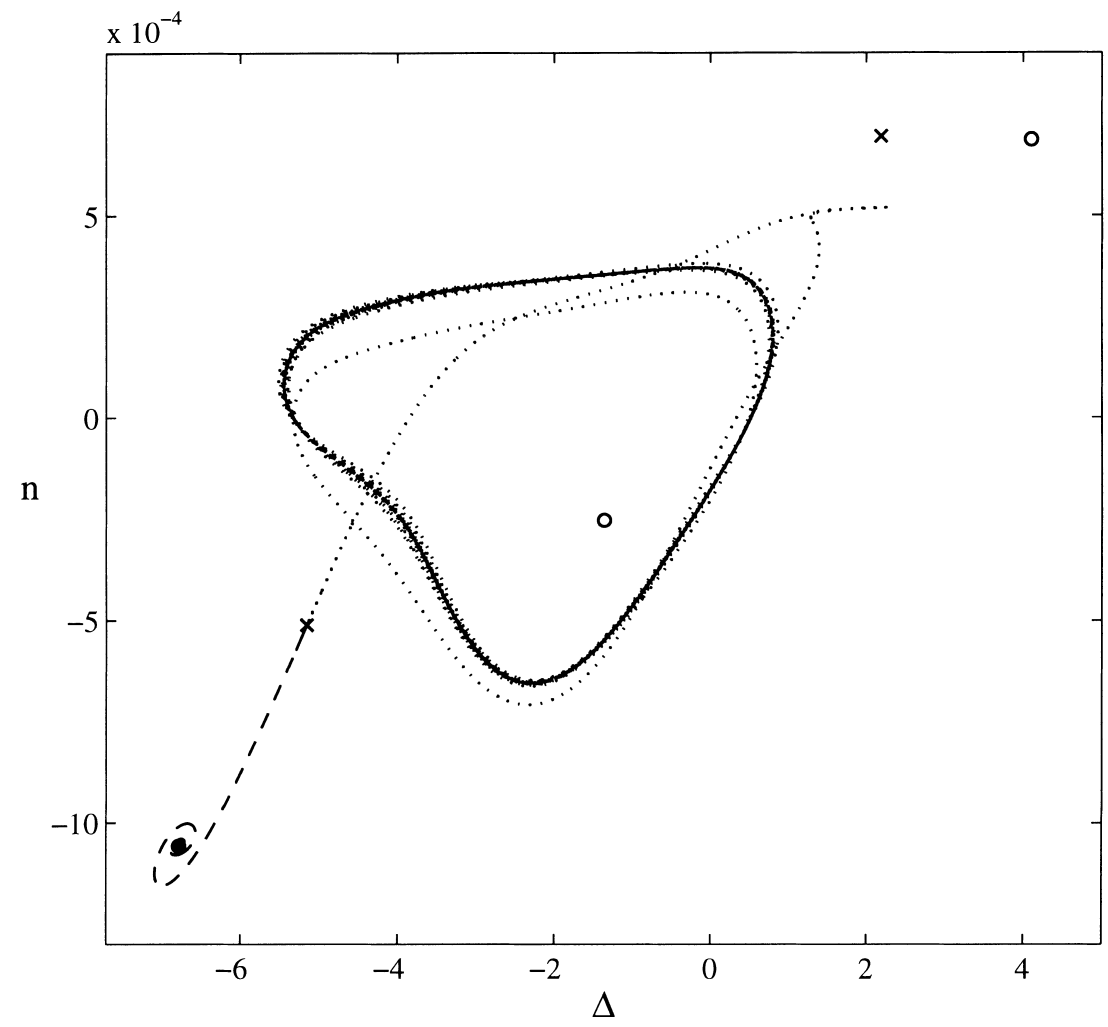

Fig. 1. Two trajectories originating in the vicinity of the saddle point on the opposite sides from the stable manifold $\left(E_{0}=E_{\mathrm{s}}, n_{0}=n_{\mathrm{s}}\right.$, and $\Delta_{0}=\Delta_{\mathrm{s}} \pm 0.005$ ). One trajectory (dashed line) is attracted to a stable fixed point which is the MGM, while the other trajectory (dotted line) converges to a limit cycle. Open circles and crosses denote the external-cavity modes and the antimodes, respectively. The feedback parameter is $\eta=0.0012$. The integration time is $50 \tau$. 


\section{Chaotic dynamics of the Lang-Kobayashi model}

We study the dynamics of the Lang-Kobayashi equations using fourth-order Adams-Bashford-Moulton predictorcorrector method [22], and view the system in the $(E, n, \Delta)$ configuration space. As an example, we consider dynamics of the system with parameter values $\alpha=6, \tau=1000, \omega_{0} \tau=-1, T=1000$, and $P=0.001$, and see how it evolves as the feedback parameter $\eta$ increases.

A large variety of chaotic transitions occurs with increasing feedback level. Each new mode with more negative $\Delta_{\mathrm{S}}$ is stable upon creation, but then becomes unstable due to the Hopf bifurcation at higher $\eta$. Fig. 1 shows two trajectories originating in the vicinity of the saddle point with initial conditions slightly displaced towards the neighboring fixed points. One of the trajectories is attracted to the stable fixed point, while the other one approaches the limit cycle that appeared after the fixed point destabilized via a Hopf bifurcation at a lower feedback level. Let us follow the evolution of this limit cycle as the feedback strength grows. First, the cycle undergoes a period-doubling bifurcation at $\eta \approx 13.25 \times 10^{-4}$ (Fig. 2(a)), and then after a cascade of bifurcations it is replaced by a chaotic attractor at $\eta=13.5 \times 10^{-4}$, as shown in Fig. 2(b).

This attractor grows in size, occupying more space between the neighboring saddle points until at $\eta \approx 14.08 \times 10^{-4}$ it merges with the attractor ruins of another ECM, which were inaccessible at a lower feedback level (Fig. 2(c)). Meanwhile the attractor gets closer to the saddle separating it from the basin of the MGM, and at $\eta \approx 14.34 \times 10^{-4}$ a boundary crisis occurs: after spending some time on the chaotic attractor, the trajectory escapes across the saddle
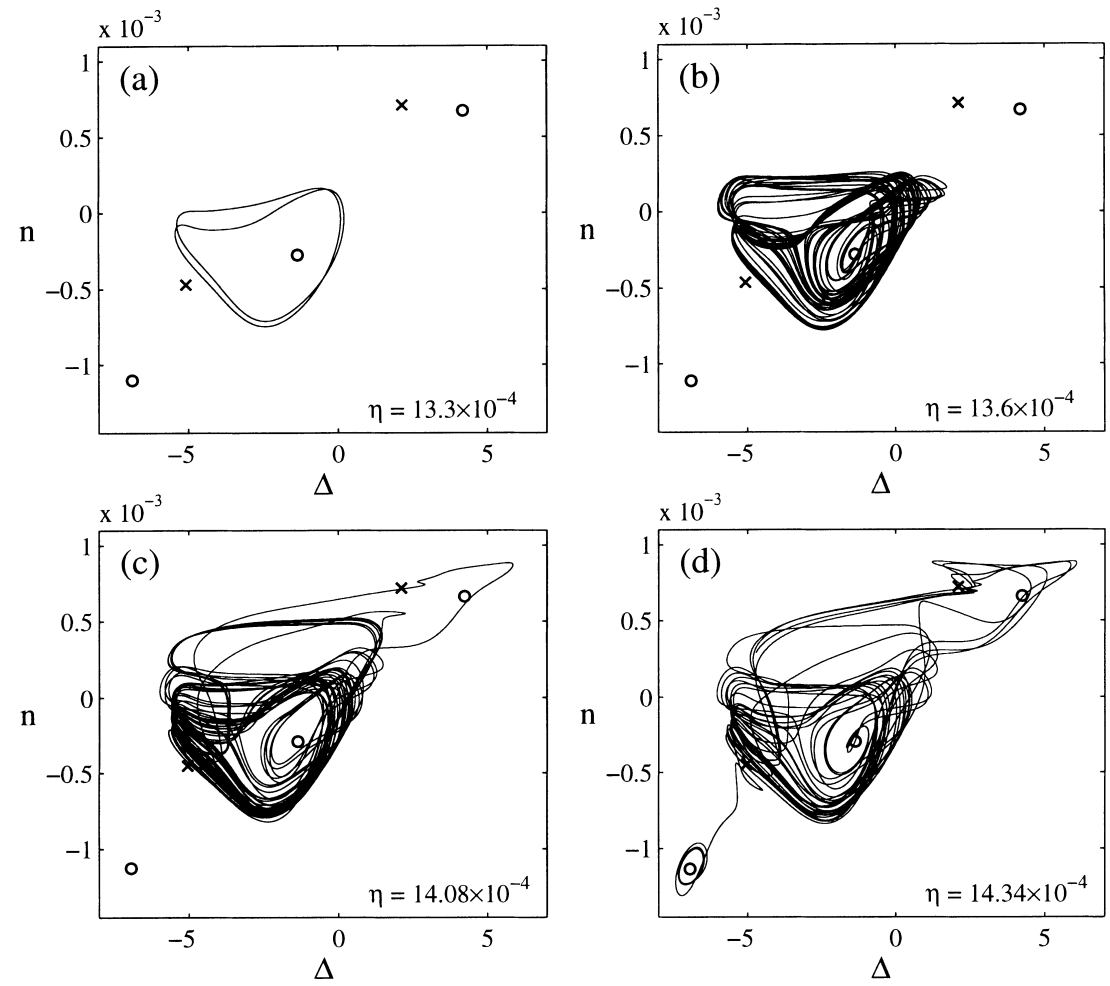

Fig. 2. Different stages in the evolution of the ECM attractor: (a) limit cycle after a period-doubling bifurcation; (b) chaotic attractor; (c) merger of the ECM attractor with attractor ruins of the neighboring ECM; (d) boundary crisis of the chaotic attractor. In all cases the integration is initialized at the ECM and the trajectory between 100 and $250 \tau$ is plotted. The duration of the transient in (d) is $207 \tau$. 
to the neighboring ECM (Fig. 2(d)). Thus, at this feedback level the chaotic behavior of the system is transient and, after a finite time interval, is replaced by a limit cycle around the MGM.

\section{Poincaré surface of section}

The sequence of transition events (stable ECM $\rightarrow$ ECM attractor $\rightarrow$ merger with lower gain attractor ruins $\rightarrow$ transient towards MGM) repeats qualitatively for higher feedback levels until a large attractor containing many ECMs is created. The LFF regime, which has been called also the Sisyphus effect [8], occurs when the system evolves on this large attractor made of the attractor ruins associated with many ECMs. We call this large attractor the Sisyphus attractor. Fig. 3 shows a Sisyphus attractor at $\eta=0.015$. One can see that the evolution of the system on the Sisyphus attractor is quite complicated. Therefore, it is convenient to characterize the system dynamics by constructing the Poincaré surface of section. Since our main focus is on transitions between attractor ruins of different ECMs, we define the Poincaré section in such a way, that it passes through all the fixed points of the system. Namely, the point $\left(E_{i}, n_{i}, \Delta_{i}\right)$ is on the surface of the section if (cf. Eq. (6))

$$
E_{i}^{2}=\frac{P-n_{i}}{1+2 n_{i}}
$$

Fig. 4 shows points $\left(\Delta_{i}, n_{i}\right)$ on the Poincaré surface of positive crossing (increasing electric field $E$ ) of the surface. The system parameters are the same as in Fig. 3. A high degree of localization around the fixed points can be seen in the phase delay coordinate, while the carrier number range of values is much broader.

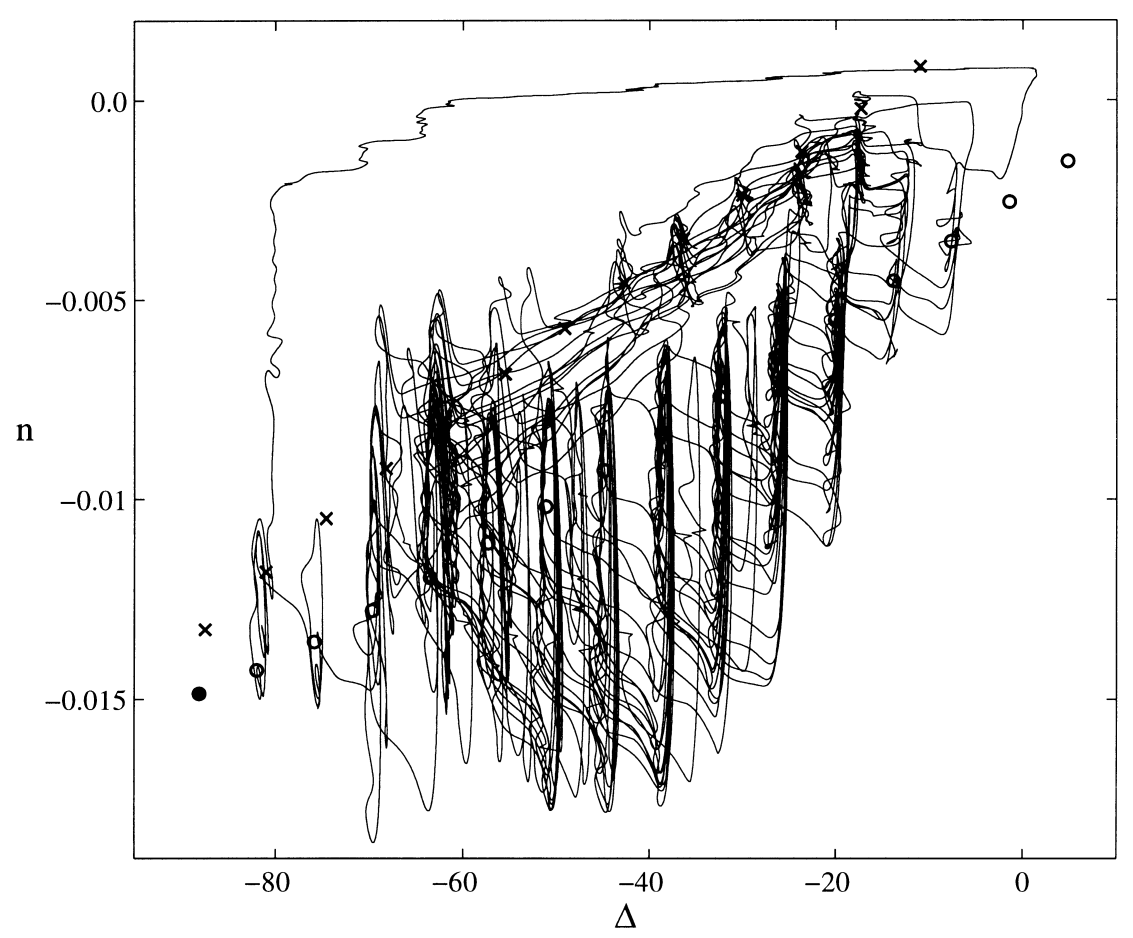

Fig. 3. Sisyphus attractor at the feedback level $\eta=0.015$. The trajectory is traced for $200 \tau$ after a $100 \tau$ pre-iteration run. The open circles denote the external-cavity modes, the crosses indicate the saddle points, and the solid circle denotes the MGM. 


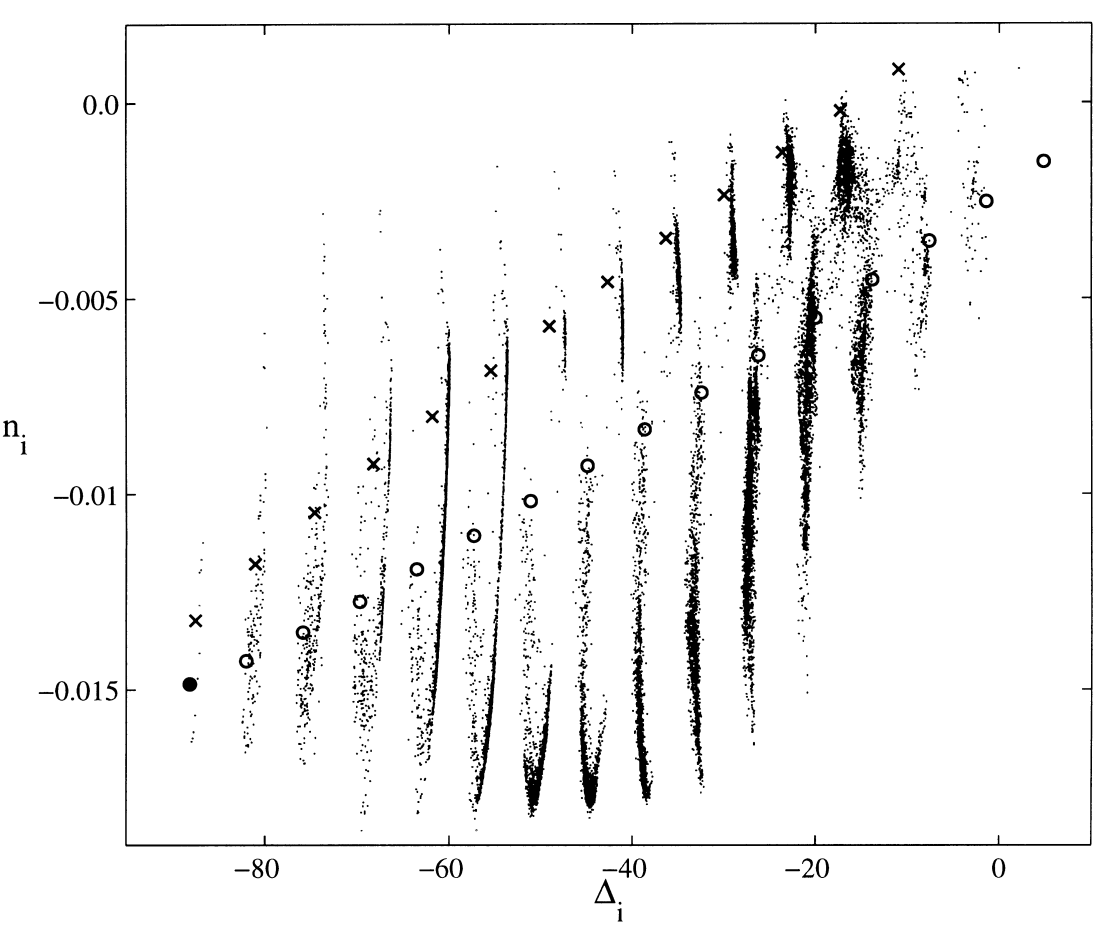

Fig. 4. Points on the Poincaré surface of section. The system parameters are the same as in Fig. 3. The plot contains 20000 points.

To study the time evolution of the system, we plot the return map of the delay coordinate $\Delta_{i+1}$ vs. $\Delta_{i}$. The result is shown in Fig. 5. Because of the localization of the delay coordinate around the fixed points, the return map shows clearly transitions of the system between different ECMs. Points along the main diagonal represent trajectories returning to the same fixed point after one loop through the three-dimensional coordinate space, while points below (above) the diagonal correspond to the transitions towards higher (lower) gain. This description of the laser dynamics allows for direct statistical analysis of system evolution, e.g., calculation of transition probability, $P_{n m}$ from $n$th to $m$ th ECM. Note that points below the diagonal are mostly confined to two closest sub-diagonals, indicating gradual evolution of the system towards MGM via nearest or next-to-nearest neighbor transitions. On the other hand, the evolution away from the MGM takes the system across 4-5 ECMs in a single loop. Note also the presence of a relatively small number of points in the upper-left corner of Fig. 5. These points indicate the special character of the transitions away from the ECMs close to the maximum gain: after the system visits these modes, both electric field magnitude and excess carrier density drop to zero, while change in the phase delay coordinate takes the system all the way to the solitary laser frequency, where it starts crossing the Poincaré surface again. We would like to stress that our ability to observe and classify transitions among the ECMs stems entirely from the particular choice of the Poincaré surface of section adopted for this study.

\section{Bifurcation analysis}

In order to study different regimes of laser operation and transitions among them, we need to understand how the system dynamics depends on the choice of the system parameters. Within the dynamical systems approach, this is usually accomplished by the construction of bifurcation diagrams. A bifurcation diagram shows what part of phase space is occupied by the system at different parameter values. Typically, the diagram is constructed by 


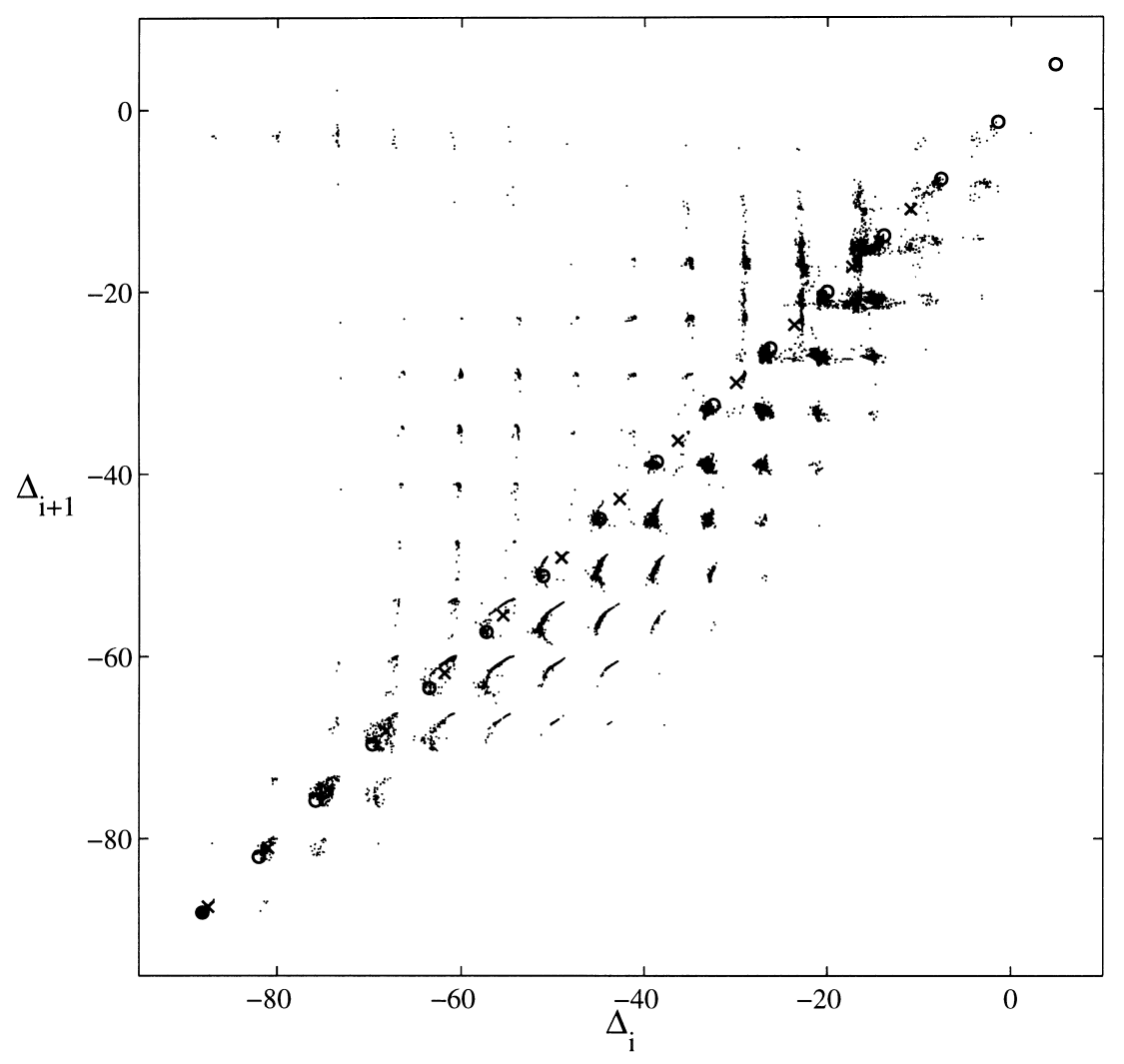

Fig. 5. Return map constructed from the points shown in Fig. 4.

plotting one of the system variables vs. a gradually varying system parameter. For systems with a simple structure (a single attractor for any fixed set of parameters), the appearance of the bifurcation diagram is not sensitive to the choice of the initial condition. However, as we have seen in Section 3, the dynamics of the system described by the Lang-Kobayashi equations may have several attractors separated by the basin boundaries. Therefore, the appearance of the bifurcation diagram depends on where the dynamics is initialized. That is, only attractors whose basins contain the initial point will appear on the bifurcation diagram.

We use this feature of the bifurcation diagram of structurally complex systems to distinguish between different types of chaotic transitions in the system. Specifically, we want to address the question of the accessibility of the MGM from the Sisyphus attractor as well as that of stability of the MGM with respect to the merger with the Sisyphus attractor. In order to answer these questions, we construct two bifurcation diagrams for a given set of parameters: first, with initial condition at the MGM, and second, starting at one of the ECMs that belongs to the Sisyphus attractor.

Fig. 6 shows the bifurcation diagram with initial condition at the MGM. The construction of the diagram is as follows. We fix parameters $\alpha, T, P, \omega_{0}, \tau$, and increase $\eta$ from zero in increments of $1.0 \times 10^{-5}$. At a given value of $\eta$ we find all stationary solutions and initialize the integration of Lang-Kobayashi equations at the MGM. After a $500 \tau$ pre-iteration run, we accumulate values of the phase delay coordinate $\Delta_{i}$ at points of intersection with the Poincaré surface during the following $2000 \tau$ and plot them in the bifurcation diagram.

Several important features of the system dynamics can be extracted from this diagram. All the modes are stable upon creation and gradually destabilize with increasing feedback level. For the first five MGMs $(\eta<0.005)$, the 


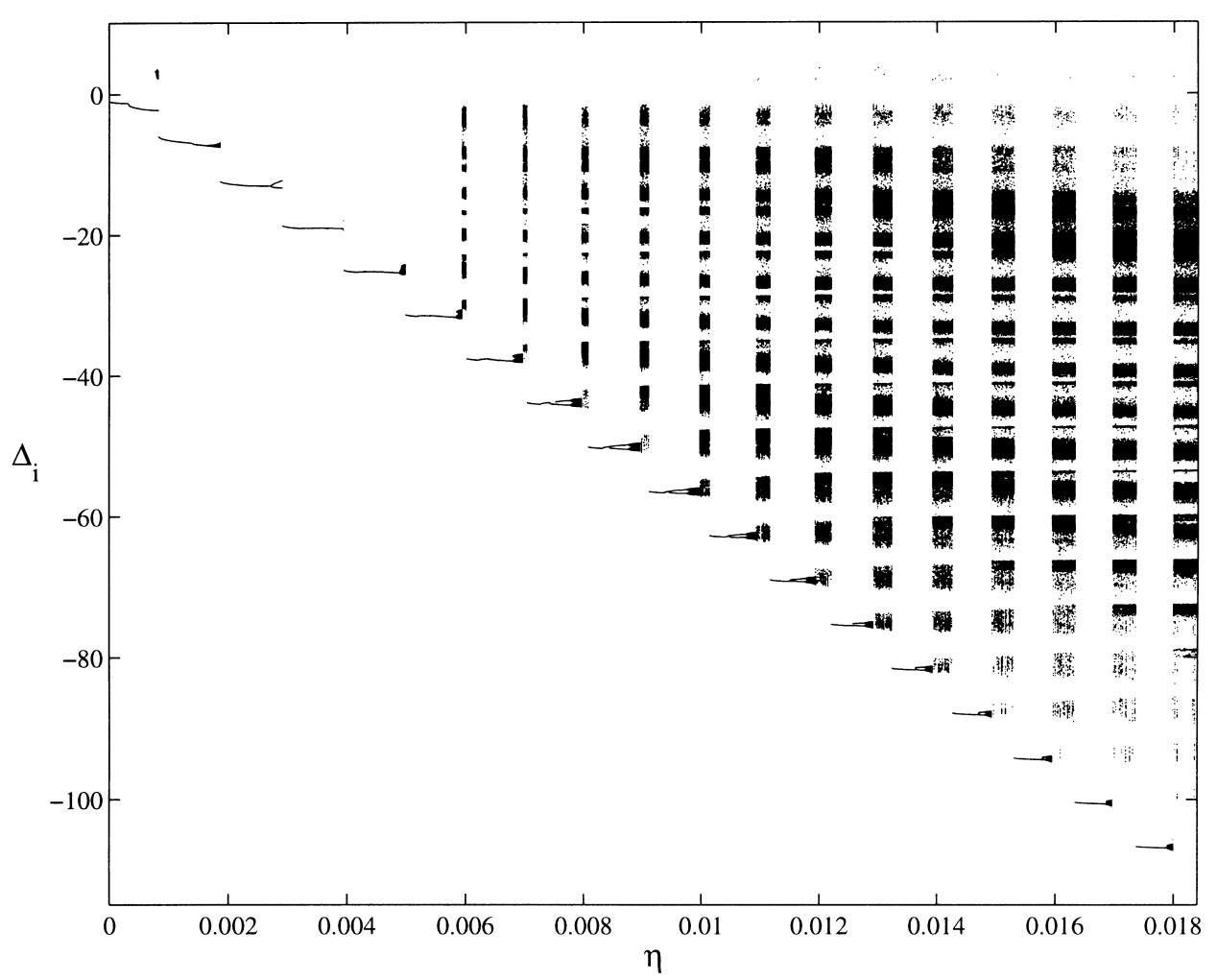

Fig. 6. Bifurcation diagram of the delay coordinate on the Poincaré surface for the dynamics initialized at the MGM (see text for details). The system parameters are $\tau=1000, \omega_{0} \tau=-1, T=1000, P=0.001$, and $\alpha=6$.

attractor is confined to the region around the mode for all values of feedback. However, starting with mode number 6, a merger of the MGM attractor with those of other modes occurs before the next mode is born. At this value of $\eta$ the Sisyphus attractor contains all ECMs. Note also that for $\eta>0.016$ the probability of visiting the MGM is very low, so that it is practically inaccessible to the Sisyphus attractor.

Information about the stability of the Sisyphus attractor with respect to the escape to a different attractor (the MGM) in the same range of parameter values can be obtained form the bifurcation diagram shown in Fig. 7, where the integration is initialized within the Sisyphus attractor. For low levels of feedback we see regions where the Sisyphus attractor collapses to a single-mode high-gain attractor. In these regions the Sisyphus effect (LFFs) is only a transient phenomenon. However, with increasing feedback, the width of the regions decreases, until the transient disappears completely for $\eta>0.008$, after which LFFs are sustained.

The same bifurcation analysis scheme can be used to explore the regimes of laser operation at different parameter values. For example, in Fig. 8 we present a bifurcation diagram for a smaller value of the linewidth enhancement factor $\alpha=4$. Note that even though the diagram looks very much like the one in Fig. 6, the interpretation of the system evolution is different, since the dynamics in Fig. 8 is initialized within the Sisyphus attractor and should be compared with that in Fig. 7. We see that, when the linewidth enhancement factor is smaller, the regions of transient LFFs persist for higher levels of feedback, alternating with the regions of sustained LFFs.

This observation suggests that the stabilization of the laser operation on the MGM is more likely for lasers with smaller values of the linewidth enhancement factor. In order to explore this dependence, we map the regimes of laser operation in the parameter plane of $\alpha$ and $\eta$. To distinguish between LFFs and stable operation on the MGM, we 


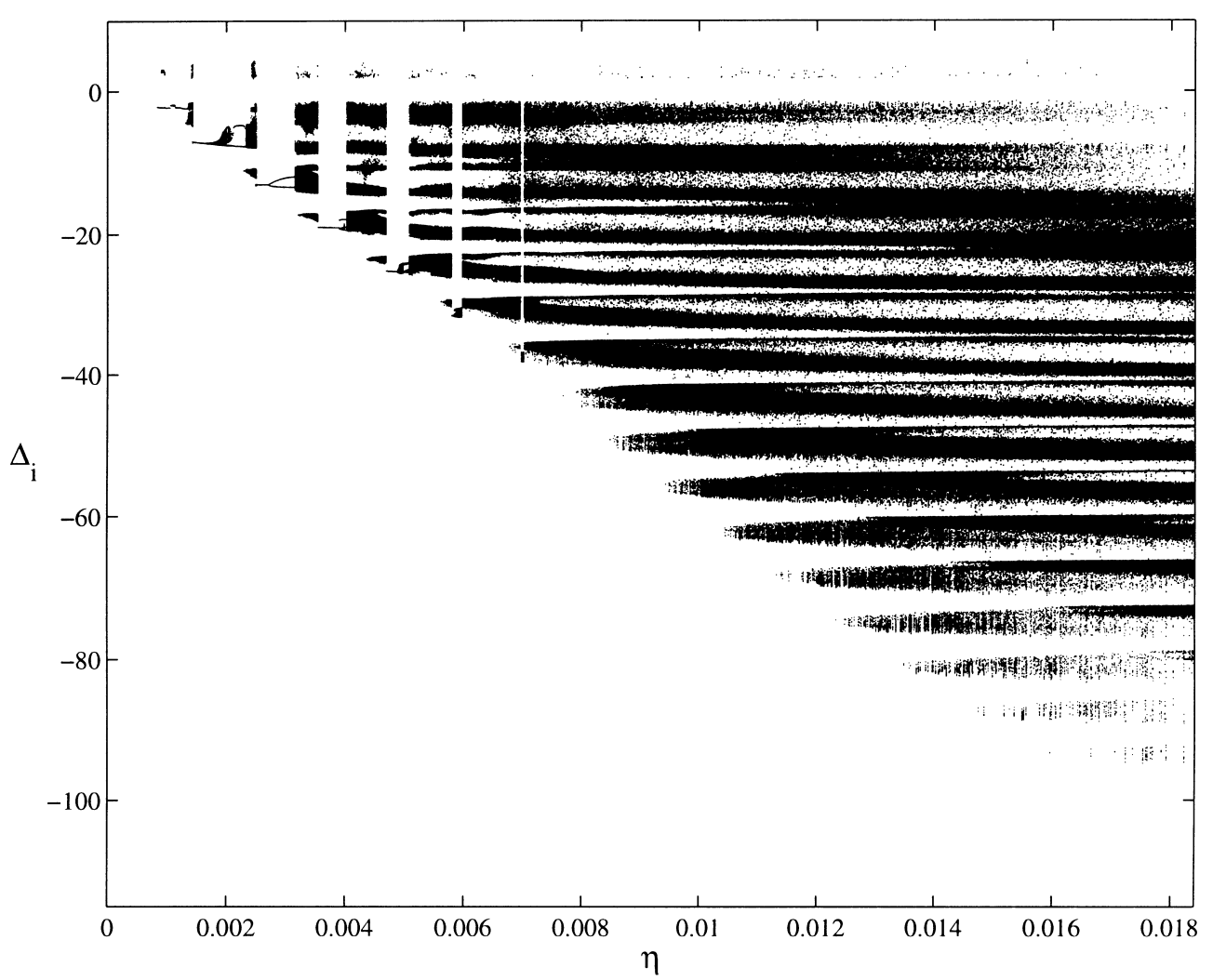

Fig. 7. Bifurcation diagram for the same parameters as in Fig. 6, but the dynamics is initialized within the Sisyphus attractor.

monitor the range of variation of the delay coordinate $\Delta_{i}$, which is large when the system evolves on the Sisyphus attractor and small when the dynamics is localized around the MGM. As a quantitative measure, we use the standard deviation

$$
\sigma_{\Delta}^{2}=\left\langle\Delta_{i}^{2}\right\rangle-\left\langle\Delta_{i}\right\rangle^{2}
$$

where the brackets denote a sample average. Fig. 9 shows in gray scale the dependence of $\sigma_{\Delta}^{2}$ on the values of $\alpha$ and normalized feedback $\eta^{\prime} \equiv \eta\left(\alpha^{2}+1\right)^{1 / 2}$. We use $\eta^{\prime}$ instead of $\eta$ in order to fix the number of available ECMs at different values of $\alpha$ (the number of available ECMs is constant along vertical lines on the plot). Depending on the value of $\alpha$, we see three qualitatively different regimes: (i) for $\alpha<3$ the system operates around the MGM for all values of feedback, (ii) for $\alpha$ increasing from 3 to about 4.5 the regions of sustained LFFs appear and grow until (iii) for $\alpha>4.5$, they merge together and the LFFs become sustained for all values of feedback. The bifurcation diagrams in Figs. 7 and 8 correspond to the regimes (iii) and (ii), respectively. Another important observation is that the Sisyphus attractor appears to be much larger in the regime (ii) than in the regime (iii), even though the same number of ECMs is available. The sharp boundary between the regions with large and small attractors suggests that we observe a transition between two structurally different Sisyphus attractors. However, the reason for the attractor size disparity in regimes (ii) and (iii) is unclear and requires further detailed exploration of the phenomenon.

We have also explored the influence of the pumping $P$ on the coexistence of the regions of transient and sustained LFFs in regime (ii). The result is shown in Fig. 10. As expected, increasing the pumping current has destabilizing 


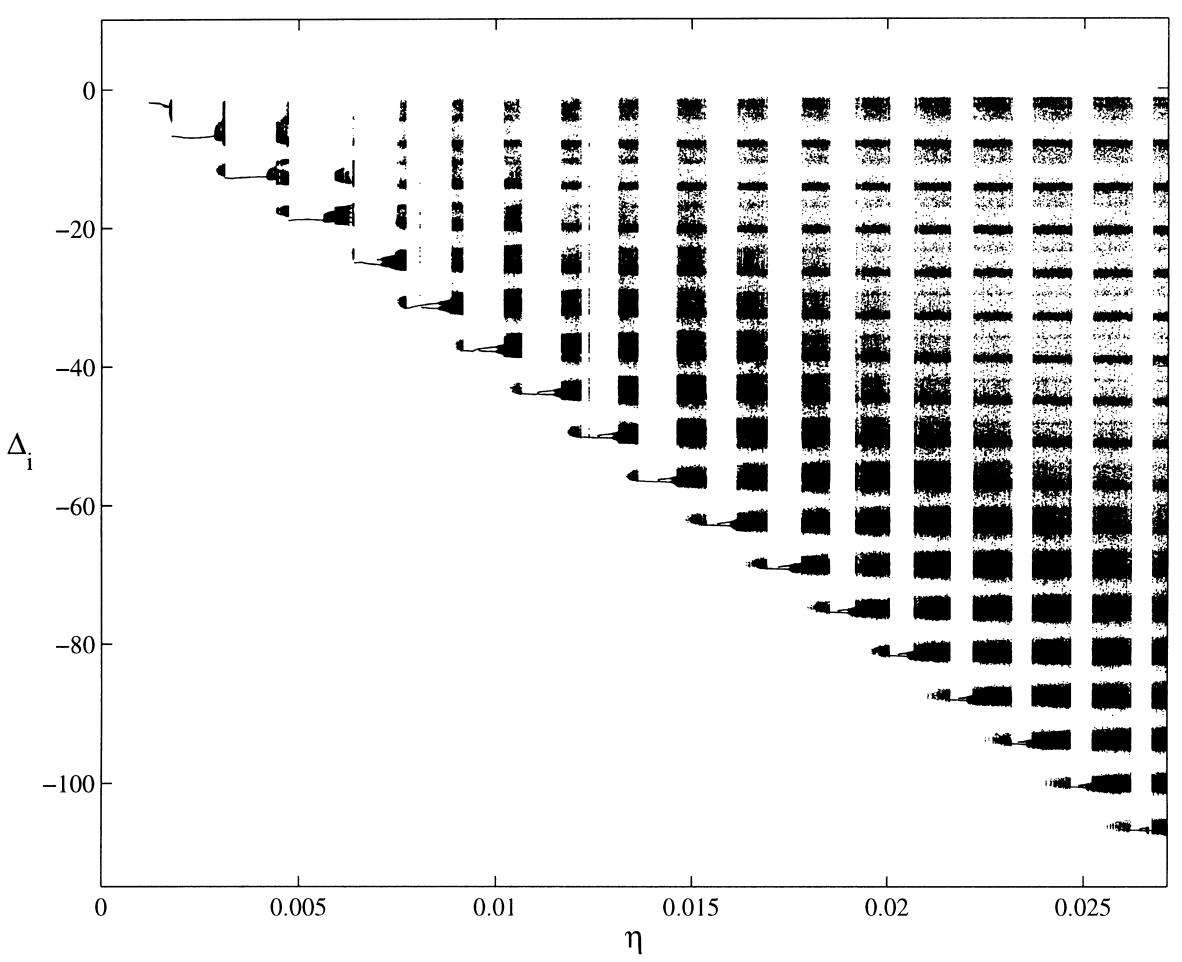

Fig. 8. Same as in Fig. 7 with a different linewidth enhancement factor $\alpha=4$.

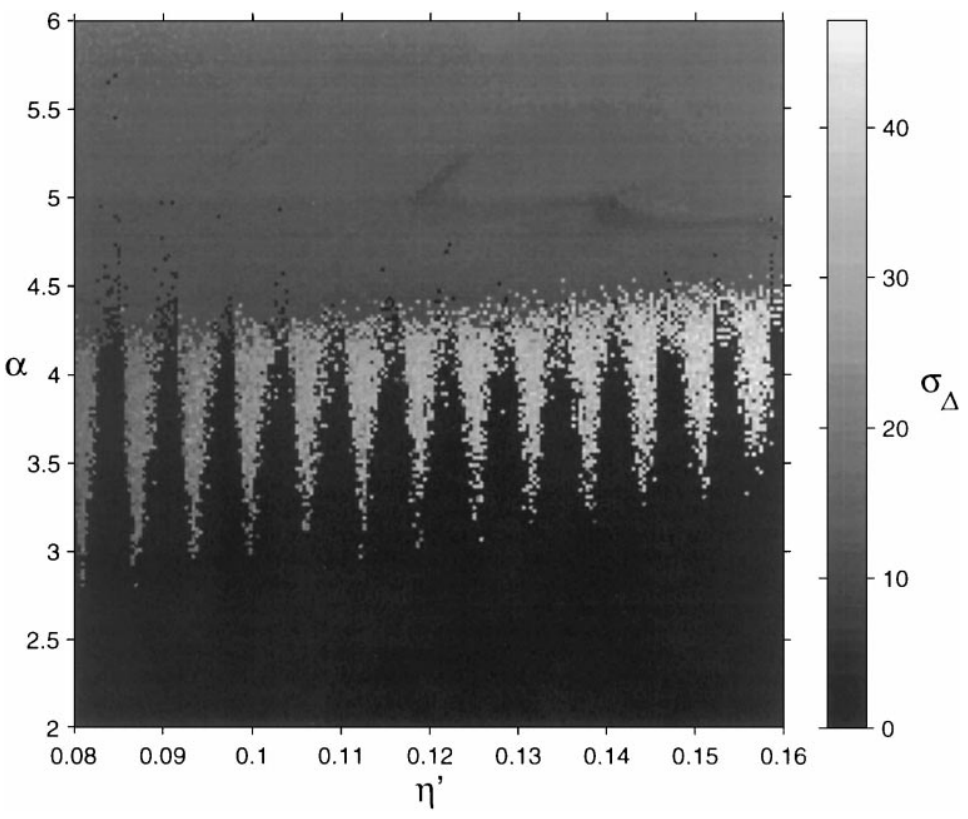

Fig. 9. Range of variation of $\Delta_{i}$ for different parameter values in the parameter plane $\alpha$ vs. $\eta^{\prime} \equiv \eta\left(\alpha^{2}+1\right)^{1 / 2}$. Other parameter values are fixed at $\tau=1000, \omega_{0} \tau=-1, T=1000$, and $P=0.001$. The standard deviation $\sigma_{\Delta}^{2}$ is calculated from a sample of $\Delta_{i}$ gathered during a $1000 \tau$ run after a $2000 \tau$ pre-iteration. 


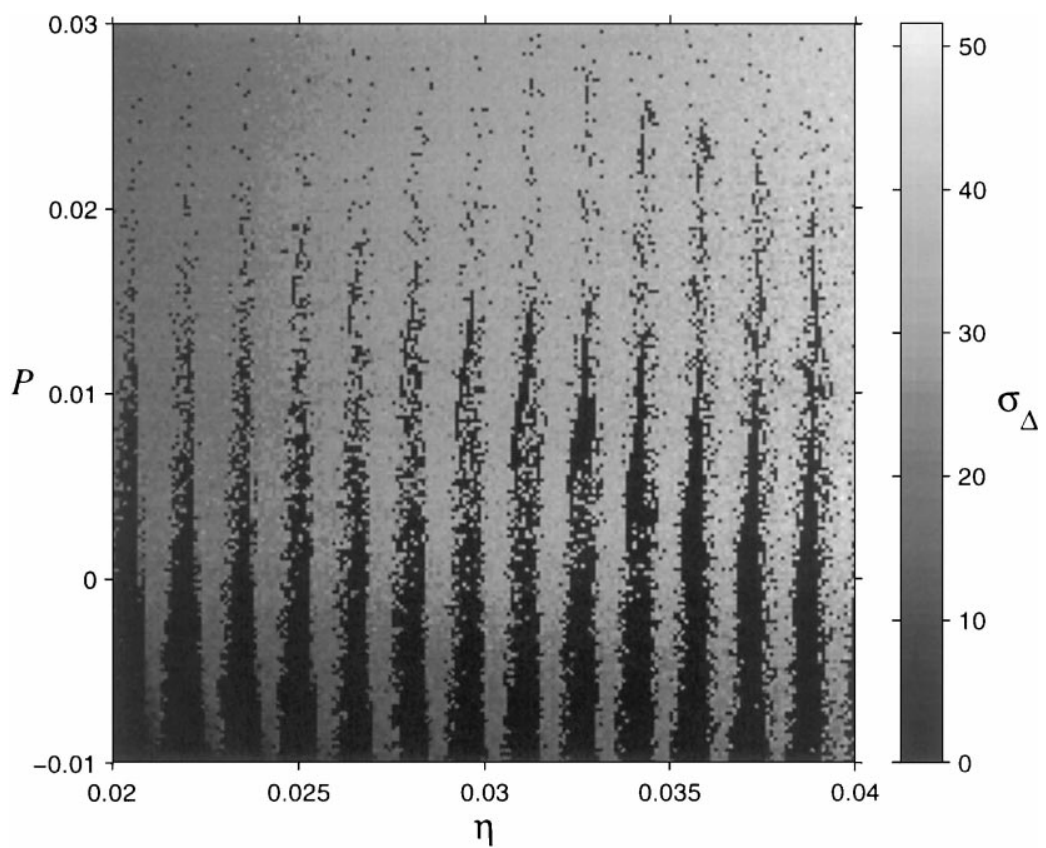

Fig. 10. Range of variation of $\Delta_{i}$ for different parameter values in the parameter plane of $P$ and $\eta$. Other parameter values are fixed at $\alpha=4, \tau=1000, \omega_{0} \tau=-1, T=1000$. The standard deviation $\sigma_{\Delta}^{2}$ is calculated from a sample of $\Delta_{i}$ gathered during a $1000 \tau$ run after a $2000 \tau$ pre-iteration.

effect on the individual ECMs, so the regions of stable emission on the MGM gradually shrink and disappear as the pumping is increased.

\section{Discussion}

We have presented a bifurcation analysis scheme that allows detailed exploration of the structure and properties of the LFF regime in a semiconductor laser with delayed optical feedback based on the Lang-Kobayashi model. The results show that LFFs can be either transient or sustained, depending on parameter values of the system. From the dynamical approach, this can be easily understood. In typical situations, the LFFs are due to wandering of trajectories of the system on a chaotic attractor, the Sisyphus attractor, that is created as the result of many crisis events that convert small chaotic attractors associated with a hierarchy of external-cavity modes into nonattracting chaotic saddles. The Sisyphus attractor is basically the dynamical union of all these chaotic saddles. The maximum gain mode, on the other hand, can be a separate attractor that coexists with the Sisyphus attractor. Depending on the basin structure of these attractors, LFFs can be either transient or sustained: they are sustained if the basins of the two attractors are completely separated; and they are transient if the Sisyphus attractor collides with the basin boundary and "invades" the basin of the attractor associated with the MGM. In the latter case, a trajectory starting from the original basin of the Sisyphus attractor typically stays near the remains of this attractor for a finite period of time and eventually asymptotes to the MGM attractor. This behavior is in good agreement with the experimental observations [13,14], where parameter regions for both transient (coexistence of LFFs and stable emission) and sustained LFFs were found. 
Exploring the dependence of the system behavior on the linewidth enhancement factor, we have observed three different regimes, with transient LFFs more prevalent for lasers with smaller $\alpha$. The reduction in "stability" of the LFFs with decreasing linewidth enhancement factor can be understood from the fact that the Sisyphus attractor exists because of the coupling between the ECMs. This coupling is induced by the large value of the linewidth enhancement factor in semiconductor lasers, and its strength decreases for smaller $\alpha$. These results are consistent with recent experiments [23] that show the reduced region in parameter space of the sustained LFFs and the broadened coexistence region (transient LFFs) for smaller values of $\alpha$.

We conclude this paper by mentioning several problems that are related to the control of semiconductor lasers. First, if LFFs are sustained, how to apply control to induce escape of a trajectory from the Sisyphus attractor to the stable emission attractor so as to eliminate the power-dropout events? Second, if the parameters of the laser are such that LFFs are only a transient phenomenon, what is the typical distribution of transient lifetime? In cases where the lifetime is extremely long, how to apply control to stabilize the laser? While there has been a tremendous amount of work on control of chaotic dynamics [24-31] and on control of chaotic diode resonators [32,33], the problem of controlling a semiconductor laser with optical feedback remains to be challenging due to the extremely high dimensionality of the system.

\section{Acknowledgements}

The first two authors were supported by AFOSR under Grant No. 749620-98-1-0400.

\section{References}

[1] C. Risch, C. Voumard, J. Appl. Phys. 48 (1977) 2083.

[2] M. Fujiwara, K. Kubota, R. Lang, Appl. Phys. Lett. 38 (1981) 217.

[3] C.H. Henry, R.F. Kazarinov, IEEE J. Quant. Electron. QE-22 (1986) 294.

[4] J. Mørk, B. Tromborg, P.L. Christiansen, IEEE J. Quant. Electron. 24 (1988) 123.

[5] J. Mørk, B. Tromborg, J. Mark, IEEE J. Quant. Electron. 28 (1992) 93.

[6] J. Sacher, et al., Phys. Rev. A 45 (1992) 1893.

[7] T. Sano, Phys. Rev. A 50 (1994) 2719.

[8] G.H.M. van Tartwijk, A.M. Levine, D. Lenstra, IEEE J. Sel. Top. Quant. Electron. 1 (1995) 466.

[9] A. Hohl, H.J.C. van der Linden, R. Roy, Opt. Lett. 20 (1995) 2396.

[10] M. Giudici, C. Green, G. Giacomelli, U. Nespolo, J.R. Tredicce, Phys. Rev. E 55 (1997) 6414.

[11] G. Vaschenko, M. Giudici, J.J. Rocca, C.S. Menoni, J.R. Tredicce, S. Balle, Phys. Rev. Lett. 81 (1998) 5536.

[12] J. Mulet, C.R. Mirasso, Phys. Rev. E 59 (1999) 5400.

[13] A. Hohl, A. Gavrielides, Phys. Rev. Lett. 82 (1999) 1148.

[14] T. Heil, I. Fischer, W. Elsäßer, , Phys. Rev. A 58 (1998) R2672.

[15] C.H. Henry, IEEE J. Quant. Electron. QE-18 (1982) 259.

[16] C. Masoller, IEEE J. Quant. Electron. 33 (1997) 796.

[17] R. Lang, K. Kobayashi, IEEE J. Quant. Electron. QE-16 (1980) 347.

[18] B. Tromborg, J. Mørk, IEEE J. Quant. Electron. 26 (1990) 642.

[19] C. Masoller, IEEE J. Quant. Electron. 33 (1997) 804.

[20] P.M. Alsing, V. Kovanis, A. Gavrielides, T. Erneux, Phys. Rev. A 53 (1996) 4429.

[21] B. Tromborg, J.H. Osmundsen, H. Olesen, IEEE J. Quant. Electron. QE-20 (1984) 1023.

[22] W.H. Beyer, CRC Standard Mathematical Tables and Formulae, 29th Edition, CRC Press, Boca Raton, FL, 1991.

[23] T. Heil, I. Fischer, W. Elsäßer, Phys. Rev. A 60 (1999) 634.

[24] E. Ott, C. Grebogi, J.A. Yorke, Phys. Rev. Lett. 64 (1990) 1196.

[25] F.J. Romeiras, C. Grebogi, E. Ott, W.P. Dayawansa, Physica D 58 (1992) 165.

[26] D. Auerbach, C. Grebogi, E. Ott, J.A. Yorke, Phys. Rev. Lett. 69 (1992) 3479.

[27] M.A. Rhode, J. Thomas, R.W. Rollins, A.J. Markworth, Phys. Rev. E 54 (1996) 4880.

[28] L. Kocarev, U. Parlitz, Phys. Rev. Lett. 77 (1996) 2206. 
[29] J.H. Peng, E.J. Ding, M. Ding, W. Yang, Phys. Rev. Lett. 76 (1996) 904.

[30] Y.-C. Lai, Phys. Rev. E 55 (1997) 4861.

[31] L. Pecora, T.L. Carroll, G. Johnson, D. Mar, Phys. Rev. E 56 (1997) 5090.

[32] T. Newell, P.M. Alsing, A. Gavrielides, V. Kovanis, Phys. Rev. Lett. 72 (1994) 1647.

[33] T. Newell, P.M. Alsing, A. Gavrielides, V. Kovanis, Phys. Rev. E 51 (1995) 2963.

[34] R.L. Davidchack, Y.-C. Lai, A. Gavrielides, V. Kovanis, Phys. Lett. A 267 (2000) 350. 